\title{
Evidence-Based Practices of English Language Teaching: A Meta-Analysis of Meta-Analyses
}

\author{
Hamad H. Alsowat ${ }^{1}$ \\ ${ }^{1}$ Taif University, Saudi Arabia \\ Correspondence: Hamad Alsowat, College of education, Taif University, P.O.Box: 888, 21944, Taif, Saudi \\ Arabia.
}

Received: September 21, 2020

Accepted: October 19, $2020 \quad$ Online Published: October 27, 2020

doi: 10.5539/elt.v13n11p75

URL: https://doi.org/10.5539/elt.v13n11p75

\begin{abstract}
This meta-analysis aims at investigating the impact of English language teaching practices on language outcomes. The literature search yielded 90 meta-analyses that were published between January 1995 and December 2019. The current study analyzed 90 meta-analyses, and these studies comprised 3496 studies, 7870 effect sizes, and nearly 700,000 students. Three moderator variables were examined: year of publication, setting, and educational level. The results showed that a) language learning strategies had a medium impact on language outcomes in general and generated the largest impact on speaking $(\mathrm{d}=0.90)$, b) technology-based language learning had a medium impact on language outcomes in general and generated the largest impact on vocabulary $(\mathrm{d}=0.98), \mathrm{c})$ explicit instruction had a medium impact on language outcomes in general and generated the largest impact on grammar $(\mathrm{d}=1.26)$, d) mobile-based language learning had a small impact on language outcomes in general and generated the largest impact on listening $(\mathrm{d}=0.73)$, and e) setting and educational level significantly moderated the impact of teaching practices on language outcomes. The findings were discussed, and implications and future research were proposed.
\end{abstract}

Keywords: English language, evidence, meta-analysis, moderators, teaching practices

\section{Introduction}

\subsection{Background}

The debate about the best practices of teaching English as a second or foreign language has been a controversial issue for decades. Although no single approach or method is effective in teaching all students, evidence-based best practices are those which promote high rates of achievement and have a record of success that is both trustworthy and valid (Gambrell et al., 2011). Although no particular approach is the best, educators claim that some teaching practices are superiors to others. Consequently, a large body of research has been conducted to explore the best practices for teaching language skills. Hundreds of meta-analyses were conducted to synthesize the effects of these practices on listening (e.g., Kang, 2015; Perez et al., 2013), speaking (e.g., Lee et al., 2014; Lin, 2014a; Wang, 2014), reading (e.g., Chaury, 2015; Hall \& Burns, 2018; Jeon \& Day, 2016; Maeng, 2014; Puzio \& Colby, 2010), writing(e.g., Chen, 2017; Kao, 2013; Kao \& Wible, 2014; Graham et al., 2015), vocabulary (e.g., Liu \& Zhang, 2018; Marulis \& Neuman, 2010; Tsai \& Tsai, 2018; Yousefi \& Biria, 2018; Yun, 2011), grammar (e.g., Alsadhan, 2011; Lee \& Huang, 2008; Shintani, 2015; Shintani et al., 2013), and language proficiency (e.g., Chiu et al., 2012; Kao, 2014; Lyster \& Saito, 2010; Sung et al., 2016; Stockard et al., 2018). These meta-analyses synthesized thousands of studies that integrated different English language teaching practices, which included explicit instruction, strategy-based instruction, skill-based instruction, technology-based learning, mobile learning, feedback, peer instruction, and other types of instruction such as textual enhancement, visual input enhancement, elicited imitation, corpus linguistics, graphic organizers, data-driven learning approach, game-based learning, within-class grouping, different gloss types, and others.

Research has little impact on students' outcomes due to the lack of "summarizing and comparing all the diverse types of evidence about what works in classrooms" (Hattie, 2009, p. 3). Therefore, existing research has to be synthesized in a way -called meta-analysis of meta-analyses- to give broader and clearer types of evidence benefiting from the massive meta-analyses research to help policymakers, parents, and teachers in the profession of language teaching and learning. Gene Glass published a journal article on meta-analysis in 1976 that laid out the essential rationale and defined many of the basic features of meta-analysis as it is known today (Schmidt \& 
Hunter, 2015). In this approach, the effect size is calculated, interpreted, and compared to other effect sizes, if necessary.

Despite the existence of massive empirical research on the impact of English language teaching practices on students' learning outcomes, the impact of those practices is limited in terms of meta-analysis of meta-analyses. Nevertheless, such reviews may lead to new types of evidence and allow educators and teachers to better understand the impact of these practices, especially when compared to each other. Therefore, this synthesis aims at identifying the impact of English language teaching practices on language outcomes (listening, speaking, reading, writing, grammar, vocabulary, and language proficiency).

\subsection{Purpose of the Study}

By synthesizing related meta-analyses, this research seeks to estimate the effect sizes of previous meta-analyses regarding the impact of different English language teaching practices on students' language outcomes. These practices will then be ranked from positive to negative to draw a clear picture of what works and what does not. It is expected to identify the high leverage English language teaching practices that focus on preparing and training English language teachers.

\subsection{English Language Teaching Practices}

Teaching the English language is a dynamic and complex task that requires adequate knowledge of the latest and effective methods to help students gain significant learning outcomes. The ELT literature covers a wide range of relatively old and up-to-date English language teaching and learning practices. To achieve the aim of this study, English language teaching practices were divided into four categories: explicit instruction, language learning strategies, technology-based language learning, and mobile-based language learning.

The four categories mentioned above guided this study as independent variables. First of all, explicit instruction is one of the most powerful tools to maximize students' outcomes. It is a direct approach that includes instructional design and delivery procedures through a series of supports or scaffolds (Archer \& Hughes, 2011). Explicit instruction is an ideal model for teaching because of its balanced approach in teaching both concepts and skills along with integrated listening, speaking, reading, writing, and vocabulary development in every lesson (Hollingsworth \& Ybarra, 2013). Elements of explicit instruction include introducing one new skill of instruction at a time, specific procedures for submitting, practicing, and reviewing skills, explicit modeling, a guided practice, providing immediate corrective feedback, ongoing review for mastery of previously taught skills, re-teaching as needed and independent practice (Rief \& Stern, 2010).

Secondly, language learning strategies help students take responsibility for their learning and promote independent and autonomous learning. They raise students' knowledge of themselves, their interests, awareness, needs and preferences, beliefs and motivation, and their strategies to develop L2 competence (White, 2008). Language learning strategies are divided into three main categories: cognitive, metacognitive, and social/affective strategies. Cognitive strategies involve the mental processing of the target language, whereas metacognitive strategies use cognitive processes to regulate the learning process. Social/affective strategies entail how learners interact with others and control themselves to enhance their learning (Benson, 2013).

Thirdly, utilizing technology in language learning has begun since its origin in the 1950s to keep up with the ongoing developments in computer technology. The development of technology in language learning results from learning theories and pedagogical considerations ( $\mathrm{Li}, 2017)$. Technology-based learning can be an engaging and interactive tool to enhance vocabulary learning, grammar, and written language skills. Using technology in the classroom encourages learners to be motivated, engaged, and responsible for their learning. Providing a source of natural language, flexible space, and immediate feedback are some advantages that make language learning a fun task (Stanley, 2013; Tafazoli \& Romero, 2017).

Fourthly, mobile technology is an emerged movement in language learning. Mobile devices such as smartphones, iPads, iPods, and tablets are becoming ubiquitous. Mobile learning enhances learner mobility in which a learner can use mobile for learning anywhere and access educational content, authentic input experiences, real-world task practice, and information without the limitations of physical location or time (Uther, 2019). Consequently, mobile learning should be included in language learning to engage with and practice the target language (Nielson, 2016).

\subsection{Synthesizing Meta-Analyses on Instruction}

Meta-analysis is defined as "the statistical analysis of a large collection of analysis results from individual studies for the purpose of integrating the findings" (Glass, 1976, p. 3). Results of the original studies are converted to one or more metrics, called "effect size", to synthesize findings from studies that use different 
measures of the same construct (Littell et al., 2008). Quantitative meta-analyses have become central to evidence-based practices in education to bridge the gap between research and practice. Evidence-based practices entail the systematic synthesis of the best evidence available to serve as the basis for best practices and as a source of best evidence in the evidence-based practice process (Sandelowski \& Barroso, 2007).

A well-known critique of meta-analysis is called "apples and oranges". It was first used in 1977 by Smith and Glass; it charges that including a diverse range of studies in a meta-analysis yields meaningless results (Card, 2012). Previous attempts to synthesize research mostly fell into this trap in analyzing meta-analyses in different disciplines with broad classifications.

Lipsey and Wilson (1993) carried out an earlier attempt to synthesize 302 studies, including 16,902 effect sizes, to provide an overview of the effects of various categories of educational, psychological, and behavioral treatments on a variety of outcomes. The mean effect size across all studies was $\mathrm{d}=0.50$. This study added a valuable contribution to the field, but it concentrated on science and math as moderators and neglected other disciplines or content areas such as social sciences and languages. Marzano (1998) conducted meta-analysis research to synthesize 143 studies with over 4,000 effect sizes and involved an estimated 1,237,000 subjects on the effect of classroom instructional techniques on achievement. He computed overall effect sizes for the knowledge domains and the cognitive, metacognitive, and self-systems. The effect sizes were $d=0.60, d=0.75$, $\mathrm{d}=0.55, \mathrm{~d}=0.74$ respectively. The overall effect size of instruction on achievement was $\mathrm{d}=0.65$. Although the independent variables were clearly defined, the outcomes covered many disciplines such as medicine, science, arts, languages, etc. Hattie (2009) synthesized a total of 816 meta-analyses, which encompassed 52,649 studies, and provided 146,626 effect sizes about the influence of some program, policy, or innovation on academic achievement in school (early childhood, elementary, high, and tertiary). The average effect size of the influence of teaching on students' achievement was $\mathrm{d}=0.42$. Unfortunately, topics of English as a second language were excluded in this synthesis.

To this end, conducting meta-analyses on instruction requires specific identification of the independent, dependent, and moderator variables to generate clear evidence that represents the actual influences of the independent and moderator variables on the dependent variables. The previous meta-analyses on English language instruction covered a wide range of the impact of various teaching practices on different language outcomes. So, there is a demand to synthesize those efforts to construct apparent and research-based foundations for language learning. Consequently, the research gap in instruction literature lies in the lack of a synthesis of meta-analyses on English language instruction on language outcomes. Therefore, this meta-analysis of meta-analyses will be conducted to seek clear evidence of English instruction on language skills.

\subsection{Research Questions}

Research Questions 1: What impact do English language teaching practices have on language outcomes?

Research Questions 2: Is the impact of English language teaching practices affected by these moderator variables (year of publication, setting, and educational level)?

\section{Method}

Researchers (e.g., Marzano, 1998; Roberts et al., 2017) stated that various considerations should be addressed when conducting informative meta-analyses. They include: (I) defining the domain of research, (2) identifying the moderator variables, (3) identifying criteria for inclusion in the study, (4) searching strategies for fugitive studies, (5) coding procedures, and (6) determining effect sizes.

\subsection{Defining the Domain of Research}

This meta-analysis aimed to compute the effect size of the previous meta-analysis studies regarding the effectiveness of English language teaching practices on language outcomes. To avoid the apples and oranges problem, categories and independent variables should be accurately defined. Teaching practices can be defined as a set of instructional methods and strategies employed in the classroom to achieve the desired teaching objectives (Khader, 2012). Based on this definition, this study considers these four independent variables as the main categories of English language teaching practices: explicit instruction, language learning strategies, technology-based language learning, and mobile-based language learning. Since the previously mentioned meta-analyses (e.g., Hattie, 2009; Lipsey \& Wilson, 1993; Marzano, 1998) did not examine language outcomes as dependent variables, this study focused on those outcomes, including listening speaking, reading, writing, vocabulary, grammar, and language proficiency. So this study did not consider dependent variables such as anxiety, thinking skills, attitudes, beliefs, participation, etc. 


\subsection{Identifying the Moderator Variables}

In qualified meta-analyses, moderator variables should be specified to estimate the relationship between the selected moderator variables and the dependent variables to attain a clear picture of the relationship between the independent variables of primary interest and the dependent variables (Marzano, 1998). Thus, moderator variables are defined as variables that modify or moderate the relationship between the independent and the dependent variables (Enders, 2012). However, due to the diversity of the meta-analyses included in this study and the large numbers of independent and dependent variables, only three moderator variables were identified: (1) year of publication, (2) setting, and (3) educational level.

\subsubsection{Year of Publication}

It was essential to notice the development of English language teaching practices in the 20th and 21st centuries. Therefore, the year of publication might influence the impact of teaching practice on language outcomes. The meta-analyses were categorized into two main categories: (1) from 1995 to 2010 and (2) from 2011 to 2019.

\subsubsection{Setting}

The setting might influence the dependent variables. For example, Mackey and Goo (2007) found that the effect of feedback was significantly greater in the EFL context than in the ESL context. Also, Jeon and Day's (2016) study showed that English as a foreign language setting (EFL)had a higher effect than English as a second language (ESL) settings. The setting was classified into: (1) EFL setting, (2) ESL setting, Mixed setting, and (4) not reported if the setting was not mentioned.

\subsubsection{Educational Level}

The third moderator variable was the educational level of the students on whom the teaching practice had been tried. The instructional technique had been undertaken. It was assumed that the effect of English language teaching practices might vary across educational levels of students. Educational levels were categorized as follow:

1) Kindergarten and Primary: grades K-6.

2) Middle and High: grades 7-12.

3) College and Adult.

4) Mixed: grades K-12 and college.

\subsection{Identifying Criteria for Inclusion in the Study}

Eligible studies for this meta-analysis should meet the inclusion criteria that were identified below.

1. The study had to be a qualitative meta-analysis.

2. It had to be published in a peer-reviewed journal or a dissertation, or a book chapter.

3. It was published within the last fifteen years, between January 1995 and December 2019.

4. It was published in the English language.

5. It focused on any English language teaching practice.

6. It focused on one or more of these language outcomes (listening, speaking, reading, writing, vocabulary, grammar, or language proficiency).

7. It reported the effect size of the practice that had been used.

\subsection{Searching Strategies for Fugitive Studies}

Three search strategies were employed to identify the studies that met the inclusion criteria: electronic search of databases, manual research, and examination of the references of the obtained studies for finding more meta-analysis studies. The electronic search included these databases and journals: ERIC, PsycINFO, Dissertation Abstracts, PsycARTICLES, EBSCO, Wiley Online Library, Taylor \& Francis, SpringerLink-Springer, Scopus-Elsevier, Sage Journals, and Google Scholar. After employing the electronic search, a hand search of the related journals was performed. References of the previous meta-analyses were searched for more secondary references. Search terms were meta-analysis*, language*, teaching*, practices*, strategy*, English*, skills* and listening* OR speaking* OR reading* OR writing* OR vocabulary* OR grammar* OR language proficiency*impact* OR effect* EFL* or ESL*. Search terms were searched as title, full text, abstract, or keywords. The search was completed on March 16, 2020, including articles published from January 1995 to December 2019. The search resulted in 835 articles. After deleting the duplicates, the search 
yielded 312 articles in English language teaching practices meta-analyses. After reading the abstracts and full-text articles, studies that did not meet the inclusion criteria were excluded. A total of 209 studies were included for the next step (Figure 1).

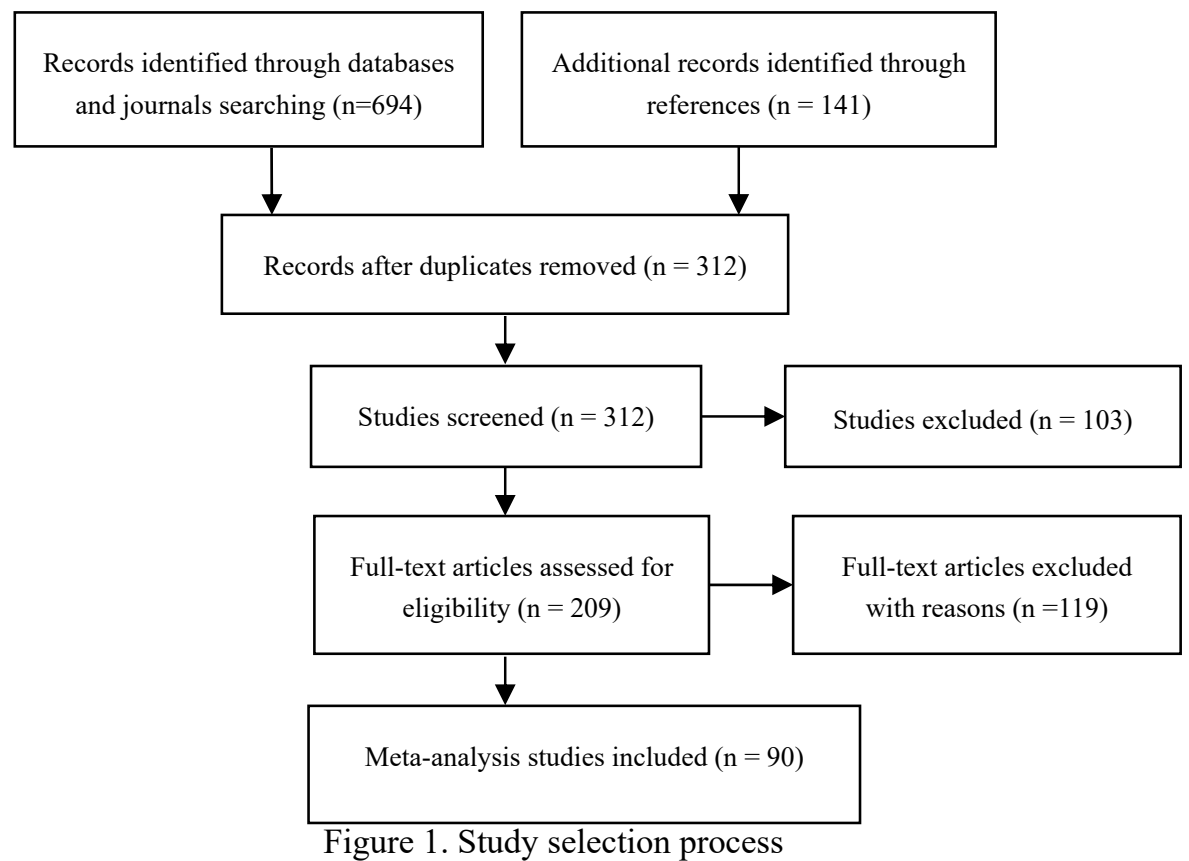

\subsection{Coding Procedures}

Eligible meta-analysis studies were coded by the author and an assistant. They were coded with respect to the independent, dependent, and moderator variables. The coding included general information of each study such as author, year of publication, setting, number of participants, educational level, number of studies included, number of effect sizes, effect sizes values. When a study had multiple independent samples, they were treated as separate studies. Based on a random sample $(30 \%)$ of the included studies, inter-rater reliability showed a Cohen's kappa of 0.92 . If there was a disagreement, the study was included for further discussion until agreement was achieved. After an agreement of $100 \%$, all studies were double coded, and the studies that did not have enough data were excluded.

The domain was coded as explicit instruction, language learning strategies, technology-based language learning, and mobile-based language learning. Explicit instruction practices were coded to sub-categories used in language teaching such as direct instruction, repeated reading, extensive reading, writing to read instruction, spelling instruction, vocabulary instruction, writing instruction, reading for writing instruction, process writing approach, processing instruction, production-based instruction, error correction and so on. Language learning strategies were classified into cognitive strategies, metacognitive strategies, affective strategies, socio-affective strategies, and types of feedback. Technology-based language learning practices were categorized into captioned videos, computer-mediated communication, synchronous computer-mediated communication, multiple media tools, online and blended learning, web-based instruction, digital game-based learning, digital tools, and computer-mediated glosses. Finally, mobile-based language learning practices were coded as teaching using iPADs, tablet PCs, cell phones, iPods, MP3 players, e-book readers, digital pens, pocket dictionaries, and classroom response systems (CRSs).

In addition, the meta-analysis studies were classified according to the dependent variables (language outcomes). The language outcomes included listening, speaking, reading, writing, grammar, vocabulary, and language proficiency. The studies were also categorized upon the moderator variables as mentioned earlier.

\subsection{Determining Effect Sizes}

Using effect size helps obtain the same metric to analyze and interpret the results of the previous meta-analyses on the impact of English language teaching practices on language outcomes. In most of the meta-analyses included in this meta-analysis, effect sizes were already calculated using either Cohen's d or Hedges' g, so no need for calculation of effect sizes. However, in a few cases, if effect sizes were not reported clearly, they were calculated using Cohen's d or Hedges' g (Glass, 1977; Hedges \& Olkin, 1985) as follows: 
Cohen's d: Effect size $=\left[\right.$ Mean $_{\text {treatment }}-$ Mean $\left._{\text {control }}\right] /$ Sigma

Sigma $=$ population standard deviation

Hodges' g: Effect size $=\left[\right.$ Mean $_{\text {treatment }}-$ Mean $\left._{\text {control }}\right] /$ SD

$\mathrm{SD}=$ sample standard deviation

For considering the meaning of the effect size, Cohen (1988) suggested $d=0.20$ was small, $d=0.50$ was medium, and $\mathrm{d}=0.80$ was large effect size. Hattie (2009) utilized an alternative way of considering the meaning of the effect size suggested by McGraw and Wong (1992), which was called the indicator of Common Language Effect (CLE). This measure was provided in all the results of this study to support interpreting the effect size.

\section{Results}

The literature search yielded 90meta-analyses that were published between January 1995 and December 2019 on the impact of English language teaching practices on language outcomes. These meta-analyses comprised 3496 studies and 7870 effect sizes. Across these studies, there were nearly 700,000 students.

Table 1. Descriptive statistics of the included meta-analyses

\begin{tabular}{llll}
\hline Categories & & $\mathrm{k}$ & $\%$ \\
\hline Publication type & Journal articles & 84 & $93.3 \%$ \\
& Dissertations & 6 & $6.7 \%$ \\
Year of publication & $1995-2010$ & 19 & $21.1 \%$ \\
& $2011-2019$ & 71 & $78.9 \%$
\end{tabular}

Table 1 indicates that most of the included meta-analyses were published in journals (93.3\%), whereas about three quarters of the meta-analyses were published from 2011 to 2019.

It is obvious that the studies published before 2010 were limited. However, the number of published studies increased and reached a peak in 2018 with 12 studies.

This meta-analysis study aimed at investigating the impact of English language teaching practices on language outcomes. To answer the first research questions, the overall effect sizes of English language teaching practices on language outcomes were computed in Table 2.

Table 2. Overall impact of the teaching practices on all types of language outcomes

\begin{tabular}{lllllll}
\hline Teaching practices & $\mathrm{k}$ & $\mathrm{n}$ & Sample & ES & CLE & Impact \\
\hline Language learning strategies & 277 & 580 & 22017 & 0.66 & $47 \%$ & Medium \\
Technology-based language learning & 649 & 988 & 110141 & 0.65 & $46 \%$ & Medium \\
Explicit instruction & 2361 & 5662 & 478287 & 0.59 & $42 \%$ & Medium \\
Mobile-based language learning & 209 & 640 & 33304 & 0.48 & $34 \%$ & Small \\
Total & 3496 & 7870 & 643749 & 0.59 & $42 \%$ & Medium \\
\hline
\end{tabular}

$\mathrm{k}=$ number of studies, $\mathrm{n}=$ number of effect sizes, $\mathrm{ES}=$ effect size

Table 2 shows that of the 7,870 effect sizes that focused on English language teaching practices, 5,662 (71.94\%) effect sizes utilized explicit instruction, 988 (12.55\%) effect sizes addressed technology-based language learning, $640(8.13 \%)$ effect sizes addressed mobile-based language learning, and $580(7.37 \%)$ addresses language learning strategies. The overall effect size across English language teaching practices was $\mathrm{d}=0.59$. Mobile-based language learning had the smallest effect size $(\mathrm{d}=0.48)$, whereas the effect sizes of the other three categories were medium and ranged from $\mathrm{d}=0.59$ for explicit instruction to $\mathrm{d}=0.66$ for language learning strategies. These findings imply the superiority of language learning strategies over other English language teaching practices on the development of language outcomes $(\mathrm{d}=0.66)$.

The impacts of all types of teaching practices; explicit instruction, language learning strategies, technology-based language learning, and mobile-based language learning were calculated as seen in Table 3 . 
Table 3. Impact of all teaching practices on language outcomes

\begin{tabular}{lllllllll}
\hline \multirow{2}{*}{ Teaching practices } & \multicolumn{2}{l}{ Language outcomes } & & & & \multirow{2}{*}{ Total } \\
\cline { 2 - 7 } & Listening & Speaking & Reading & Writing & Grammar & Vocabulary & Proficiency & \\
\hline Language strategies & - & $\mathbf{0 . 9 0}$ & 0.61 & 0.86 & 0.06 & 0.71 & 0.59 & 0.66 \\
Technology-based learning & 0.74 & 0.39 & 0.33 & 0.54 & - & $\mathbf{0 . 9 8}$ & 0.70 & 0.65 \\
Explicit instruction & 0.51 & 0.84 & 0.47 & 0.51 & $\mathbf{1 . 2 6}$ & 0.90 & 0.58 & 0.59 \\
Mobile-based learning & $\mathbf{0 . 7 3}$ & 0.50 & 0.30 & 0.25 & - & 0.49 & 0.57 & 0.48 \\
Total & 0.69 & 0.77 & 0.46 & 0.54 & $\mathbf{1 . 2 4}$ & 0.87 & 0.60 & 0.59 \\
\hline
\end{tabular}

Table 3 illustrates that the highest impact of language learning strategies was observed in speaking $(\mathrm{d}=0.90)$, which was higher than the impact on writing $(\mathrm{d}=0.86)$ and vocabulary $(\mathrm{d}=0.71)$. Language learning strategies were less effective in teaching language proficiency $(\mathrm{d}=0.59)$ and grammar $(\mathrm{d}=0.06)$. The overall impact of language learning strategies on language skills was medium $(\mathrm{d}=0.66)$. In addition, the highest impact of technology-based language learning was observed in vocabulary $(\mathrm{d}=0.98)$, which was higher than the impact on listening $(\mathrm{d}=0.74)$ and language proficiency $(\mathrm{d}=0.70)$. Technology-based language learning was less effective in teaching speaking $(\mathrm{d}=0.39)$ and reading $(\mathrm{d}=0.33)$. The overall impact of technology-based language learning on language skills was medium $(\mathrm{d}=0.65)$. Moreover, the highest impact of explicit instruction was observed in grammar $(\mathrm{d}=1.26)$ which was higher than the impact on vocabulary $(\mathrm{d}=0.90)$ and speaking $(\mathrm{d}=0.84)$. Explicit instruction was less effective in teaching listening $(\mathrm{d}=0.51)$ and reading $(\mathrm{d}=0.47)$. The overall impact of explicit instruction on language skills was medium $(\mathrm{d}=0.59)$. Finally, the highest impact of mobile-based language learning was observed in listening $(\mathrm{d}=0.73)$, which was higher than the impact on language proficiency $(\mathrm{d}=0.57)$ and speaking $(\mathrm{d}=0.50)$. Mobile-based language learning was less effective in teaching vocabulary $(\mathrm{d}=.49)$, reading $(\mathrm{d}=0.30)$ and writing $(\mathrm{d}=0.25)$. The overall impact of mobile-based language learning on language skills was small $(\mathrm{d}=0.48)$. Table 3 also illustrates that the highest impact of teaching practices was on grammar $(\mathrm{d}=1.24)$ which was higher than the impact on vocabulary $(\mathrm{d}=0.87)$ and speaking $(\mathrm{d}=0.77)$. English language teaching practices were less effective in teaching writing $(\mathrm{d}=0.54)$ and reading $(\mathrm{d}=0.46)$.

Table 4 to Table 10 presented the impact of different types of English language teaching practices on language outcomes, including listening, speaking, reading, writing, grammar, vocabulary, and language proficiency. For each outcome, the teaching practices were arranged according to the value of the effect size.

Table 4. Impact of teaching practices on listening

\begin{tabular}{lllllll}
\hline Teaching practices & $\mathrm{k}$ & $\mathrm{n}$ & Sample & $\mathrm{ES}$ & $\mathrm{CLE}$ & Impact \\
\hline Graphic organizers & 4 & 4 & 398 & 1.39 & $99 \%$ & Large \\
Captioned videos & 15 & 15 & 1071 & 0.99 & $71 \%$ & Large \\
Mobile devices & 4 & 5 & 208 & 0.73 & $51 \%$ & Medium \\
Multiple media tools & 50 & 78 & 4330 & 0.69 & $49 \%$ & Medium \\
Language learning strategies & 13 & 19 & 955 & 0.32 & $23 \%$ & Small \\
Total & 86 & 121 & 6962 & 0.69 & $49 \%$ & Medium \\
\hline
\end{tabular}

$\mathrm{k}=$ number of studies, $\mathrm{n}=$ number of effect sizes, $\mathrm{ES}=$ effect size

Table 4 indicates that using graphic organizers had a large effect size when teaching listening $(d=1.39, n=4)$. Consequently, graphic organizers were the best practice in teaching listening skills. Captioned videos also had a large effect size $(\mathrm{d}=0.99, \mathrm{n}=15)$. Other teaching practices had small to medium effect sizes on listening skills. The overall effect size of the teaching practices on listening was medium $(\mathrm{d}=0.69, \mathrm{n}=121)$. 
Table 5. Impact of teaching practices on speaking

\begin{tabular}{lllllll}
\hline Teaching practices & $\mathrm{k}$ & $\mathrm{n}$ & Sample & ES & CLE & Impact \\
\hline Language learning strategies & 9 & 13 & 517 & 0.90 & $64 \%$ & Large \\
Peer-peer interaction & 9 & 25 & 2782 & 0.89 & $64 \%$ & Large \\
Skill-based instruction & 86 & 170 & 3298 & 0.86 & $61 \%$ & Large \\
Mobile devices & 6 & 11 & 569 & 0.50 & $36 \%$ & Medium \\
Peer-mediated learning & 9 & 13 & 1474 & 0.49 & $35 \%$ & Small \\
Computer-mediated communication & 25 & 25 & 2615 & 0.40 & $29 \%$ & Small \\
Synchronous computer-mediated communication & 5 & 11 & 186 & 0.39 & $28 \%$ & Small \\
Total & 149 & 268 & 11441 & 0.77 & $55 \%$ & Medium \\
\hline
\end{tabular}

$\mathrm{k}=$ number of studies, $\mathrm{n}=$ number of effect sizes, $\mathrm{ES}=$ effect size

For teaching speaking skills, Table 5 shows a large effect size of language learning strategies, peer-peer interaction, and skill-based Instruction on the development of speaking skills. The effect sizes were $(\mathrm{d}=0.90$, $\mathrm{n}=13 ; \mathrm{d}=0.89, \mathrm{n}=25 ; \mathrm{d}=0.86, \mathrm{n}=170)$ respectively. Mobile devices had a medium effect size $(\mathrm{d}=0.50, \mathrm{n}=11)$, whereas the other teaching practices had a small effect size on speaking ranging from $d=0.39$ to $d=0.49$. The overall effect size of the teaching practices on speaking was medium $(\mathrm{d}=0.77, \mathrm{n}=268)$.

Table 6. Impact of teaching practices on reading

\begin{tabular}{lllllll}
\hline Teaching practices & $\mathrm{k}$ & $\mathrm{n}$ & Sample & $\mathrm{ES}$ & $\mathrm{CLE}$ & Impact \\
\hline Graphic organizers & 18 & 31 & 3100 & 0.82 & $59 \%$ & Large \\
Computer-mediated glosses & 9 & 11 & 366 & 0.73 & $52 \%$ & Medium \\
Language learning strategies & 90 & 283 & 6927 & 0.61 & $44 \%$ & Medium \\
Extensive reading & 83 & 114 & 8842 & 0.57 & $41 \%$ & Medium \\
Joint book reading & 29 & 29 & 3410 & 0.55 & $39 \%$ & Medium \\
Small-group reading interventions & 26 & 27 & 2600 & 0.54 & $39 \%$ & Medium \\
CALL & 12 & 18 & 875 & 0.53 & $38 \%$ & Medium \\
L1 glosses & 67 & 21 & 20330 & 0.52 & $37 \%$ & Medium \\
Direct instruction & 249 & 1896 & 14961 & 0.51 & $36 \%$ & Medium \\
Digital tools & 20 & 89 & 4024 & 0.49 & $35 \%$ & Small \\
Peer-mediated learning & 22 & 28 & 2221 & 0.47 & $34 \%$ & Small \\
Skill-based instruction & 151 & 161 & 15248 & 0.46 & $33 \%$ & Small \\
Reading interventions & 37 & 93 & 8862 & 0.41 & $29 \%$ & Small \\
Balancing reading instruction & 47 & 82 & 22753 & 0.38 & $27 \%$ & Small \\
Comprehension strategy instruction & 141 & 361 & 22926 & 0.32 & $23 \%$ & Small \\
Mobile devices & 64 & 121 & 11338 & 0.30 & $21 \%$ & Small \\
Within class grouping & 15 & 51 & 5410 & 0.22 & $16 \%$ & Small \\
Synchronous computer-mediated communication & 3 & 7 & 118 & 0.21 & $15 \%$ & Small \\
Reading media & 38 & 58 & 171055 & 0.21 & $15 \%$ & Small \\
Education technology & 85 & 85 & 60721 & 0.16 & $11 \%$ & Small \\
Computer-assisted instruction & 17 & 40 & 1590 & 0.13 & $9 \%$ & Small \\
Total & 1223 & 3606 & 387677 & 0.46 & $33 \%$ & Small \\
\hline
\end{tabular}

$\mathrm{k}=$ number of studies, $\mathrm{n}=$ number of effect sizes, $\mathrm{ES}=$ effect size

Table 6 shows that reading had the majority of meta-analyses. This was because of the importance of reading skills among the other language skills. Using graphic organizers was the only teaching practice with a large effect on reading $(\mathrm{d}=0.82, \mathrm{n}=31)$. Teaching practices such as computer-mediated glosses, language learning strategies, extensive reading, Joint book reading, small-group reading interventions, CALL, L1 glosses, and direct instruction had a medium effect size on reading skills ranging from $d=0.51$ to $d=0.73$. The remaining teaching practices had very small to small effect sizes on reading skills, ranging from $d=0.13$ to $d=0.49$. The overall effect size of the teaching practices on reading was small $(\mathrm{d}=0.46, \mathrm{n}=3606)$. 
Table 7. Impact of teaching practices on writing

\begin{tabular}{|c|c|c|c|c|c|c|}
\hline Teaching practices & $\mathrm{k}$ & $\mathrm{n}$ & Sample & ES & CLE & Impact \\
\hline Educational technology applications & 16 & 21 & 1650 & 0.93 & $66 \%$ & Large \\
\hline Graphic organizers & 9 & 18 & 2173 & 0.88 & $63 \%$ & Large \\
\hline Language learning strategies & 52 & 101 & 6488 & 0.86 & $61 \%$ & Large \\
\hline Types of feedback & 175 & 228 & 21571 & 0.64 & $46 \%$ & Medium \\
\hline Spelling instruction & 36 & 40 & 6037 & 0.58 & $41 \%$ & Medium \\
\hline Reading for writing instruction & 102 & 167 & 35472 & 0.50 & $36 \%$ & Medium \\
\hline Peer-mediated learning & 24 & 28 & 3174 & 0.49 & $35 \%$ & Small \\
\hline Computers & 29 & 29 & 2900 & 0.45 & $32 \%$ & Small \\
\hline Writing strategy instruction & 238 & 307 & 20002 & 0.44 & $31 \%$ & Small \\
\hline Process writing approach & 24 & 24 & 7865 & 0.34 & $24 \%$ & Small \\
\hline Technology-based instruction & 6 & 11 & 620 & 0.28 & $20 \%$ & Small \\
\hline Error correction & 17 & 32 & 1378 & 0.26 & $19 \%$ & Small \\
\hline Mobile devices & 7 & 28 & 1456 & 0.25 & $18 \%$ & Small \\
\hline Synchronous computer-mediated communication & 5 & 8 & 227 & 0.16 & $11 \%$ & Small \\
\hline Total & 740 & 1042 & 111013 & 0.54 & $39 \%$ & Medium \\
\hline
\end{tabular}

$\mathrm{k}=$ number of studies, $\mathrm{n}=$ number of effect sizes, ES = effect size

As Table 7 indicates, writing had the third total of effects sizes included in the meta-analyses studies $(n=1042)$. Educational technology applications, graphic organizers and language learning strategies had large effect sizes on writing development $(\mathrm{d}=0.93, \mathrm{n}=21 ; \mathrm{d}=0.88, \mathrm{n}=18 ; \mathrm{d}=0.86, \mathrm{n}=101)$ respectively. Only three practices had medium effect sizes on writing: feedback, spelling instruction, and reading for writing instruction; their effect sizes ranged from $\mathrm{d}=0.50$ to $\mathrm{d}=0.64$. Other teaching practices had very small to small effect sizes on writing skills ranging from $\mathrm{d}=0.16$ to $\mathrm{d}=0.49$. The overall effect size of the teaching practices on writing was medium $(\mathrm{d}=0.54, \mathrm{n}=1042)$.

Table 8. Impact of teaching practices on grammar

\begin{tabular}{lllllll}
\hline Teaching practices & $\mathrm{k}$ & $\mathrm{n}$ & Sample & $\mathrm{ES}$ & CLE & Impact \\
\hline Processing instruction & 33 & 79 & 2301 & 2.33 & $166 \%$ & Large \\
Comprehension-based instruction & 30 & 80 & 2677 & 1.84 & $131 \%$ & Large \\
Textual enhancement & 39 & 51 & 3900 & 0.71 & $51 \%$ & Medium \\
Graphic organizers & 2 & 4 & 398 & 0.68 & $49 \%$ & Medium \\
Production-based instruction & 59 & 113 & 6638 & 0.56 & $40 \%$ & Medium \\
Visual input enhancement & 16 & 20 & 1257 & 0.22 & $16 \%$ & Small \\
Language learning strategies & 4 & 5 & 326 & 0.06 & $4 \%$ & Small \\
Total & 183 & 352 & 17497 & 1.24 & $89 \%$ & Large \\
\hline
\end{tabular}

$\mathrm{k}=$ number of studies, $\mathrm{n}=$ number of effect sizes, $\mathrm{ES}=$ effect size

For teaching grammar, Table 8 shows a large effect size of processing instruction and comprehension-based instruction on teaching grammar; $(\mathrm{d}=2.33, \mathrm{n}=79 ; \mathrm{d}=1.84, \mathrm{n}=1.84)$. Textual enhancement, graphic organizers and production-based instruction had medium effect sizes; $(\mathrm{d}=0.71, \mathrm{n}=51 ; \mathrm{d}=0.68, \mathrm{n}=4 ; \mathrm{d}=0.56, \mathrm{n}=113)$ respectively. Visual input enhancement had a small effect size on grammar $(\mathrm{d}=0.22, \mathrm{n}=20)$. Unlike other skills, language learning strategies had a very small size on grammar $(d=0.06, n=5)$. The overall effect size of the teaching practices on grammar was large $(\mathrm{d}=1.24, \mathrm{n}=352)$.

Table 9. Impact of teaching practices on vocabulary

\begin{tabular}{lllllll}
\hline Teaching practices & $\mathrm{k}$ & $\mathrm{n}$ & Sample & ES & CLE & Impact \\
\hline Computer-mediated glosses & 6 & 6 & 199 & 1.40 & $100 \%$ & Large \\
Extensive reading & 33 & 35 & 2552 & 1.02 & $73 \%$ & Large \\
Digital games learning & 36 & 179 & 2446 & 0.99 & $71 \%$ & Large \\
Vocabulary instruction & 157 & 380 & 11874 & 0.92 & $66 \%$ & Large \\
Captioned videos & 10 & 10 & 714 & 0.87 & $62 \%$ & Large \\
Different gloss types & 66 & 101 & 5928 & 0.83 & $59 \%$ & Large \\
Computer-assisted instruction & 16 & 16 & 1684 & 0.75 & $54 \%$ & Medium \\
Graphic organizers & 5 & 5 & 583 & 0.73 & $52 \%$ & Medium \\
Language learning strategies & 24 & 36 & 1314 & 0.71 & $51 \%$ & Medium \\
Mobile devices & 43 & 84 & 4074 & 0.49 & $35 \%$ & Small \\
Reading interventions & 3 & 9 & 2009 & 0.38 & $27 \%$ & Small \\
Total & 399 & 861 & 33377 & 0.87 & $62 \%$ & Large \\
\hline
\end{tabular}

$\mathrm{k}=$ number of studies, $\mathrm{n}=$ number of effect sizes, $\mathrm{ES}=$ effect size 
Table 9 points out that teaching vocabulary gained a high interest of researchers $(n=861)$. Many teaching practices had large effect sizes on vocabulary teaching, such as computer-mediated glosses, extensive reading, digital games learning, vocabulary instruction, captioned videos, and different gloss types ranging from $\mathrm{d}=0.83$ to $d=1.40$. Computer-assisted instruction and graphic organizers had medium effect sizes $(d=0.75, n=16 ; d=0.73$, $\mathrm{n}=5)$ respectively. On the other hand, mobile devices $(\mathrm{d}=0.49, \mathrm{n}=84)$ and reading interventions $(\mathrm{d}=0.38, \mathrm{n}=9)$ had only small effect sizes on vocabulary. The overall effect size of the teaching practices on vocabulary was large $(\mathrm{d}=0.87, \mathrm{n}=861)$.

Table 10. Impact of teaching practices on language proficiency

\begin{tabular}{lllllll}
\hline Teaching practices & $\mathrm{k}$ & $\mathrm{n}$ & Sample & $\mathrm{ES}$ & $\mathrm{CLE}$ & Impact \\
\hline Elicited imitation & 24 & 21 & 1089 & 1.34 & $96 \%$ & Large \\
Corpus linguistics tools & 78 & 178 & 4288 & 0.92 & $66 \%$ & Large \\
Technology applications & 90 & 90 & 6781 & 0.85 & $61 \%$ & Large \\
Computer technology & 37 & 52 & 3980 & 0.85 & $61 \%$ & Large \\
Information and communication technology & 9 & 29 & 1045 & 0.81 & $58 \%$ & Large \\
Oral feedback & 34 & 34 & 827 & 0.74 & $53 \%$ & Medium \\
Digital game-based learning & 72 & 77 & 6664 & 0.73 & $52 \%$ & Medium \\
Web-based instruction & 31 & 31 & 3414 & 0.67 & $48 \%$ & Medium \\
Language learning strategies & 98 & 142 & 6445 & 0.59 & $42 \%$ & Medium \\
Mobile devices & 85 & 391 & 15659 & 0.57 & $41 \%$ & Medium \\
Direct instruction & 59 & 299 & 19680 & 0.54 & $39 \%$ & Medium \\
Shared book reading & 54 & 226 & 3989 & 0.28 & $20 \%$ & Small \\
Online and blended learning & 45 & 50 & 1921 & 0.19 & $14 \%$ & Small \\
Total & 716 & 1620 & 75782 & 0.60 & $43 \%$ & Medium \\
\hline
\end{tabular}

$\mathrm{k}=$ number of studies, $\mathrm{n}=$ number of effect sizes, $\mathrm{ES}=$ effect size

Table 10 shows a large effect size of elicited imitation, corpus linguistics tools, technology applications, computer technology, and information and communication technology to develop language proficiency. Their effect sizes were $(\mathrm{d}=1.34, \mathrm{n}=21 ; \mathrm{d}=0.92, \mathrm{n}=178 ; \mathrm{d}=0.85, \mathrm{n}=90 ; \mathrm{d}=0.85, \mathrm{n}=52 ; \mathrm{d}=0.81, \mathrm{n}=29)$ respectively. Oral feedback, digital game-based learning, web-based instruction, language learning strategies, mobile devices, and direct instruction had medium effect sizes on language proficiency which ranged from $d=0.54$ to $d=0.74$. Shared book reading had a small effect size $(\mathrm{d}=0.28, \mathrm{n}=226)$, and online and blended learning had a very small size $(\mathrm{d}=0.19, \mathrm{n}=50)$ on language proficiency. The overall effect size of the teaching practices on language proficiency was medium $(\mathrm{d}=0.60, \mathrm{n}=1620)$.

To answer the second research question, regarded the moderator variables that might affect English language teaching practices, these moderators: year of publication, setting, and educational level were computed as seen in Table 11 .

Table 11. Effects of moderator variables on language outcomes

\begin{tabular}{|c|c|c|c|c|c|c|}
\hline Moderator variables & $\mathrm{k}$ & $\mathrm{n}$ & ES & Std. Deviation & Std. Error Mean & $\mathrm{P}$ value \\
\hline \multicolumn{7}{|l|}{ Year of publication } \\
\hline $1995-2010$ & 705 & 6121 & 0.60 & 0.29 & 0.061 & \multirow{2}{*}{0.36} \\
\hline 2010-2019 & 2791 & 1749 & 0.67 & 0.32 & 0.037 & \\
\hline \multicolumn{7}{|l|}{ Setting } \\
\hline EFL & 499 & 1249 & 0.71 & 0.27 & 0.058 & \multirow{4}{*}{$<0.01$} \\
\hline ESL & 376 & 968 & 0.41 & 0.24 & 0.052 & \\
\hline Mixed & 661 & 1817 & 0.63 & 0.38 & 0.084 & \\
\hline Not reported & 1960 & 3836 & 0.62 & 0.33 & 0.053 & \\
\hline \multicolumn{7}{|l|}{ Educational level } \\
\hline Kindergarten/Primary & 260 & 468 & 0.49 & 0.99 & 0.210 & \multirow{4}{*}{$<0.01$} \\
\hline Middle/High & 678 & 1572 & 0.54 & 0.46 & 0.070 & \\
\hline College/Adult & 462 & 933 & 0.85 & 0.61 & 0.107 & \\
\hline Mixed & 2096 & 4897 & 0.63 & 0.27 & 0.037 & \\
\hline
\end{tabular}

$\mathrm{k}=$ number of studies, $\mathrm{n}=$ number of effect sizes, $\mathrm{ES}=$ effect size 
The moderator variable of the year of publication was coded to two periods: from 1995 to 2010 and from 2010 to 2019. Table 11 shows that the effect size of the first period was $d=0.60$ and $d=0.67$ for the second period. This difference was not statistically significant. The setting moderator was coded to EFL, ESL, Mixed, and Not reported. English language teaching practices used in EFL contexts had a larger effect size $\mathrm{d}=0.71$ than other settings. This difference was statistically significant $(\mathrm{p}<0.01)$. The smallest effect size was seen in ESL contexts, $\mathrm{d}=0.41$. The educational level moderator was coded to Kindergarten/Primary, Middle/High, College/Adult, and Mixed. The largest effect size was for college and adult students $\mathrm{d}=0.85$. This difference was also statistically significant $(\mathrm{p}<0.01)$. The smallest effect size of the English language teaching practices was observed when teaching kindergarten and primary students $\mathrm{d}=0.49$.

\section{Discussion}

\subsection{What Impact Do English Language Teaching Practices Have on Language Outcomes}

The findings of this meta-analysis show an overall medium effect size of English language teaching practices on language outcomes. Language learning strategies have the largest effect size on language outcomes since they are purposeful mental actions that the learner creatively employs to meet learning-related needs, used consciously, and can be taught to help learners to develop self-regulation, complete L2 tasks, and move toward L2 proficiency (Oxford et al., 2018). When learners are encouraged to use cognitive, metacognitive, and sociocognitive strategies, they begin to think, plan, monitor, and evaluate their learning. Consequently, they become independent learners and take responsibility for their learning. This finding is consistent with Hattie (2009), who concluded that the largest effect size on achievement was noticed in areas of metacognition. Furthermore, the productive skills -speaking and writing-are highly affected by using language learning strategies. Research has revealed that successful L2 writers have metacognitive knowledge about themselves as writers, the characteristics of the writing task, and the appropriate strategies for accomplishing their writing purpose (Oxford, 2013). Therefore, learners should be supported to develop metacognitive habits of mind to control and develop engagement and motivation to speak an L2 (Goh, 2018). On the other hand, language learning strategies have almost no impact on grammar due to the ignorance of grammar learning strategies in the cognitive strategy category, so grammar learning strategies obtain the least concern and attention of any area of language learning strategies (Oxford, 2017).

Technology-based language learning also has a medium effect size on language outcomes since technology adds flexibility and variability to second language education and adds avenues for monitoring, supporting the learning process, and hence it supports and scaffolds language learning (Zhao, 2005). For the success of technology in language learning, it is essential to use the appropriate tools and look for effective ways to integrate technology into the curriculum. For example, technology is more effective in teaching vocabulary than other skills. This influence might be because technology can construct various situations where a particular word is used. Multimedia materials also facilitate the acquisition of new words $(\mathrm{Li}, 2017)$. The previous research included in the meta-analysis does not review the effect of technology-based learning on grammar teaching. This might be due to the nature of grammar teaching and the tendency of teachers to use inductive and deductive approaches in teaching grammar.

Although there are hundreds of studies on the impact of explicit instruction on language outcomes, its effect size is not large because it is a teacher-centered approach that teachers' characteristics can influence. Nevertheless, the findings show that explicit instruction is highly effective in teaching grammar, vocabulary, and speaking. Its high effectiveness in teaching grammar refers to the fact that "most English and foreign language textbooks use a style of explicit grammar teaching called deductive instruction, in which different structures are presented and then practiced in different kinds of exercises and activities" (Cowan, 2008, pp. 31-32). This finding is consistent with the study of Norris and Ortega (2000), which revealed that explicit instruction was more effective than implicit instruction. Another reason for the large effect of explicit instruction on grammar is that explicit instruction plays an essential role in most classrooms (Ur, 2012). Furthermore, explicit instruction is essential for frequent words of any L2 because they are requisites for language use. Accordingly, explicit teaching is necessary for effective vocabulary teaching (Schmitt, 2000). Moreover, explicit instruction has a large effect on speaking since it gives a teacher a chance to teach speaking through systematic and clear stages that allow students to acquire the skill easily.

Mobile-based language learning has become visible in the last decade, but it is not quite effective in developing overall language outcomes, although it is moderately effective in some aspects of language outcomes. Listening is the skill that received the highest impact of mobile learning, but this finding does not give clear evidence due to the small number of effect sizes being computed. Language proficiency gains the highest number of effect sizes, but the effect size is still medium. This shows that mobile learning needs more effective delivery methods to have a high impact on language proficiency. Writing skills do not benefit from mobile learning since writing 
is a skill that requires guidance from teachers. Unfortunately, previous research does not consider exploring the effect of mobile-based language learning on grammar for unknown reasons.

In common, the total effect size of all teaching practices on language outcomes indicates a high impact on grammar and vocabulary only. This might be referred to as the easiness of teaching these sub-skills and the tendency of teachers to concentrate on grammar and vocabulary as essential aspects of language learning. On the other hand, the effect of teaching practices on writing and reading is still low since teaching these skills is complicated due to the complex abilities of writing and reading skills.

For the effect of teaching practices on each language outcome, many meta-analyses were conducted to synthesize the impact of English language teaching practices on listening. Graphic organizers have a large impact on listening, although there are only a few effect sizes. They are effective because they provide learners with a meaningful framework to relate new information to existing cognitive structures (Kansizoglu, 2017). Captioned videos also have a large effect on listening; captioning is proposed as a mediating device that visually assists the learner where the listening skill falls short (Perez et al., 2013). Mobile devices have a medium impact on listening skills because listening requires long-term training, and $90 \%$ of the interventions included were shorter than six months (Sung et al., 2015). Similarly, multiple media tools such as audiocassette players, video, radio, laboratories are moderately effective since they assist L2 listening practices, promote motivation, and access authentic listening materials (Kang, 2015). Although the high impact of language learning strategies on some skills such as speaking, reading, and writing, they have a small effect on listening. An explanation of this small impact may be that listening strategies may be an exception to this tendency because these strategies are rarely taught in the L2 (Plonsky, 2011).

Regarding speaking skills, the overall effect size of language teaching practices is medium. Only two meta-analyses synthesized the impact of language learning strategies which obtained the largest effect size on speaking. These strategies are taught purposely to help students acquire speaking skills. Peer-peer instruction and skill-based instruction also have large effect sizes on speaking. Skilled-based instruction also has a large effect size on speaking. The studies that addressed skill-based instructions recruit very small samples and lack diversity, especially in terms of different ages (Lee et al., 2015), so more expensive studies are needed to clarify the effect of skill-based instruction on speaking. Mobile devices have a medium effect size on speaking, but these findings are limited due to the absence of meta-analyses that synthesized the impact of mobile devices on speaking as the only dependent variable. Peer-mediated learning, computer-mediated communication, and synchronous computer-mediated communication have small effect sizes on speaking. Meta-analyses that utilized those techniques are limited, and previous primary studies on those methods have shown mixed and sometimes contradictory results (Lin, 2014a). Another reason for this finding may refer to the lack of meta-analyses that only aim to synthesize these methods' effects on speaking skills.

About half of the included meta-analyses were conducted in the field of reading teaching practices. This shows the importance of reading skills and a large number of attempts to obtain effective teaching practices. Still, the overall effect size of the different teaching practices on reading skills is disappointing due to the difficulty of teaching this skill and its association with other basic skills as prerequisites. Graphic organizers have a high impact on reading, and this may be referring to the fact that students who use graphic organizers become strategic learners and critical thinkers. Also, they help students pay attention to keywords, key ideas, and the relation between them (Kansizoglu, 2017). Other teaching practices such as computer-mediated glosses, language learning strategies, extensive reading, joint book reading, small-group reading interventions, CALL, L1 glosses, and direct instruction have a medium impact on reading. The common feature of these teaching practices is their capabilities in giving students a chance to be active learners. They also include using two or more techniques concurrently, such as computer and glosses, explicit and implicit instruction, L1 glosses, and the target language. Teachers should be aware of using some practices when teaching reading, such as mobile devices, synchronous computer-mediated communication, reading media, education technology, and computer-assisted instruction since other scaffolding teaching practices should support these practices.

Teaching writing has gained more interest in the last decade. Researchers have tried various approaches and methods to improve writing skills. Although educational technology applications are not often used in teaching writing, they have proven to improve writing skills. This may be due to the collaborative engagement and involvement and their ability to enhance students' online correction and feedback. Graphic organizers and language learning strategies also significantly impact students' writing because they enhance strategic awareness and help students become independent learners. However, other teaching practices have small to medium effect sizes on writing, so teachers should be careful when using them and integrate them with other methods to be more effective in teaching writing skills.

Few meta-analyses were conducted on grammar teaching. The overall effect size of grammar teaching practices is large, especially processing instruction and comprehension-based instruction. Processing instruction helps 
students "overcome several processing principles that define their attentional priorities and prevent them from paying attention to specific grammatical features in the input" (Shintani et al., 2013). Comprehension-based instruction also assists students when introducing new grammatical rules. Grammar instruction can be more effective if it entails a combination of comprehension-based and production-based activities. Visual input enhancement and language learning strategies are less effective in teaching grammar due to their unusual use and the few meta-analyses conducted on grammar.

Vocabulary instruction is associated with an overall large effect size. Most teaching practices are highly effective in teaching vocabulary. Computer and technology-based practices are commonly used in this field. For example, students learn more vocabulary when they are exposed to computer-mediated text glosses. In addition, digital game-based vocabulary learning is superior to other approaches, but the game design can moderate the effects of digital game-based learning on vocabulary. Game design is challenging when using game-based learning since adventure-based games are more attractive, motivating, and stimulating than non-adventure-based games (Chen et al., 2018). Mobile devices are still a new development in vocabulary learning, so more studies are necessary to draw a clear picture of their impact on vocabulary learning.

Regarding language proficiency, many meta-analyses were conducted to explore the impact of different teaching practices on language proficiency. The overall effect size of these practices is medium. Elicited imitation, corpus linguistics tools, technology applications, computer technology, and information and communication technology were of large effect sizes on language proficiency. It is noticed that the number of studies included for these practices is small. This indicates a need for more meta-analyses to emphasize these findings. Some moderators affect the impact of these practices, such as age, context, and publication type. Oral feedback, digital game-based learning, web-based instruction, language learning strategies, mobile devices, and direct instruction were of medium effect size on language proficiency. The previous meta-analyses suggested additional assumptions to raise the effectiveness of these practices, such as scaffolding teaching and students' participation.

\subsection{Is the Impact of English Language Teaching Practices Affected by These Moderator Variables (Year of Publication, Setting, and Educational Level)}

The year of publication does not moderate the effectiveness of English language teaching practices while setting and educational level moderate the effectiveness of English language teaching practices. Meta-analyses that have been conducted from 2011 to 2019 have a higher impact. Although the difference was not significant, it may refer to the recent development of English language teaching practices though these meta-analyses included several almost old primary studies. The setting in which teaching practices are perfumed significantly affects the language outcomes. The findings reveal that teaching practices in EFL contexts gain a higher impact than those of ESL or mixed settings even though ESL settings should be better situations to practice teaching practices than EFL settings. This finding is consistent with Mackey and Goo (2007) and Jeon and Day (2016), which indicated that EFL settings had a higher effect than ESL. This finding needs to be interpreted with attention since most of the included meta-analyses $(56 \%)$ did not report setting as a moderator variable. Finally, the educational level moderator significantly affects the English language outcomes. Teaching practices allocated for college and adult students have a higher impact than others. This may refer to adult students' mental and psychological maturity and their ability to take responsibility for their learning. For the aim of generalizability, these results should be treated with considerable caution since $60 \%$ of the included meta-analyses have mixed students, from kindergarten to college level.

\subsection{Limitations}

The findings of this meta-analysis have some limitations that should be considered. First, the findings concerning the impact of the teaching practices cannot be generalized to other domains such as mathematics, science, medicine, or engineering. Second, due to the different types of language outcome; listening, speaking, reading, writing, grammar, vocabulary, and language proficiency in most of the included meta-analyses, it is difficult to aggregate the effect size of specific teaching practice on a specified outcome due to the number of effect sizes and treatments in each meta-analysis. Third, since this meta-analysis is a synthesis of previous meta-analyses, it is difficult to find sufficient information to determine exact effect sizes, such as missing data of the setting or the educational level of the students. Fourth, publication bias should be considered since the inclusion criteria may affect selecting the included meta-analyses. Moreover, the quality of the included meta-analyses may affect the findings since the quality of the included studies has not been asserted. The findings of this study should consider the limitations of meta-analysis since generalization is merely related to the characteristics of the included meta-analyses.

\subsection{Conclusion and Future Research}

This meta-analysis provides an overall positive medium impact of English language practices on language outcomes. The effect sizes of sub-categories teaching practices vary from small to large, depending on the types 
of teaching practices and language outcomes. Language learning strategies are superior to other practices, especially in teaching speaking and writing. It is suggested to do more research in this area to investigate the benefits of language learning strategies and the factors that assist teachers in adopting this method. Explicit instruction is very effective in teaching grammar and vocabulary due to its powerful influences on sub-skills. Technology use is favored in introducing new vocabulary, but it should go along with other practices to scaffold teaching. Therefore, this field of study should attract more attention to gain deep insight into incorporating technology in language learning/teaching. On the other hand, mobile technology has begun to attract the attention of English language practitioners and researchers, but the small magnitude of the previous meta-analyses does not provide clear evidence of its capability to improve language skills. Utilizing teaching practices for grammar, vocabulary, and listening gain less research interest. Hence, future meta-analyses should bridge this research gap by investigating this area's best language teaching practices.

\section{References}

References marked with an asterisk indicate studies included in the meta-analysis.

*Abraham, L. (2008). Computer-mediated glosses in second language reading comprehension and vocabulary learning: A meta-analysis. Computer Assisted Language Learning, 21(3), 199-226. https://doi.org/10.1080/09588220802090246

*Alsadhan, R. O. (2011). Effect of textual enhancement and explicit rule presentation on the noticing and acquisition of L2 grammatical structures: A meta-analysis [Master, thesis, Colorado State University]. CSU Theses and Dissertations. Retrieved from https://mountainscholar.org/handle/10217/48103

Archer, A., \& Hughes, C. (2011). Explicit instruction: Effective and efficient teaching. The Guilford Press.

*Ardasheva, Y., Wang, Z., Adesope, O., \& Valentine, J. (2017). Exploring effectiveness and moderators of language learning strategy instruction on second language and self-regulated learning outcomes. Review of Educational Research, 87(3), 544-582. https://doi.org/10.3102/0034654316689135

Benson, P. (2013). Teaching and researching: Autonomy in language learning (2nd ed.). Routledge. https://doi.org/10.4324/9781315833767

*Bernard, R., Borokhovski, E., Schmid, R., Tamim, R., \& Abrami, P. (2014). A meta-analysis of blended learning and technology use in higher education: from the general to the applied. Journal of Computing in Higher Education, 26, 87-122. https://doi.org/10.1007/s12528-013-9077-3

*Biber, D., Nekrasova, T., \& Horn, B. (2011). The effectiveness of feedback for L1-English and L2 writing development: A meta-analysis. TOEFL iBT Research Report No. TOEFLiBT-14. Educational Testing Service. https://doi.org/10.1002/j.2333-8504.2011.tb02241.x

*Boulton, A., \& Cobb, T. (2017). Corpus use in language learning: A meta-analysis. Language Learning, 67(2), 348-393. https://doi.org/10.1111/lang.12224

*Brobst, P. (2009). A meta analysis of studies on error correction in second language writing [Unpublished master's thesis]. Colorado State University.

*Bus, A. G., van Ijzendoorn, M. H., \& Pellegrini, A. D. (1995). Joint book reading makes for success in learning to read: A meta-analysis on intergenerational transmission of literacy. Review of Educational Research, 65(1), 1-21. https://doi.org/10.3102/00346543065001001

Card, N. (2012). Applied meta-analysis for social science research. Guilford Press.

*Chang, M.-M., \& Lin, M.-C. (2013). Strategy-oriented web-based English instruction: A meta-analysis. Australasian Journal of Educational Technology, 29(2), 203-216. https://doi.org/10.14742/ajet.67

*Chaury, P. (2015). The effects of strategy instruction on reading comprehension in English as a foreign language. Concordia Working Papers in Applied Linguistics, 6, 2-25.

*Chen, M., Tseng, W., \& Hsiao, T. (2018). The effectiveness of digital game-based vocabulary learning: A framework-based view of meta-analysis. British Journal of Educational Technology, 49(1), 69-77. https://doi.org/10.1111/bjet.12526

*Chen, T. (2017). Technology-supported peer feedback in ESL/EFL writing classes: a research synthesis. Computer Assisted Language Learning, 29(2), 365-397. https://doi.org/10.1080/09588221.2014.960942

*Cheung, A. C., \& Slavin, R. E. (2011). The effectiveness of education technology for enhancing reading achievement: A meta-analysis. Center for Research and Reform in Education.

${ }^{*}$ Chiu, Y. H. (2013). Computer-assisted second language vocabulary instruction: A meta-analysis. British Journal of Educational Technology, 44(2), 52-56. https://doi.org/10.1111/j.1467-8535.2012.01342.x 
*Chiu, Y., Kao, C., \& Reynolds, B. (2012). The relative effectiveness of digital game-based learning types in English as a foreign language setting: A meta-analysis. British Journal of Educational Technology, 43(4), 104-107. https://doi.org/10.1111/j.1467-8535.2012.01295.x

*Cho, K., Lee, S., Joo, M-H., \& Becker, B. (2018). The effects of using mobile devices on student achievement in language learning: A meta-analysis. Education Sciences, 8(3), 1-16. https://doi.org/10.3390/educsci8030105

${ }^{*}$ Cobb, M. (2010). Meta-analysis of the effectiveness of task-based interaction in form-focused instruction of adult learners in foreign and second language teaching [Doctoral dissertation, The University of San Francisco]. Retrieved from https://repository.usfca.edu/cgi/viewcontent.cgi?article=1389\&context=diss

Cohen, J. (1988). Statistical power analysis for the behavioral sciences (2nd ed.). L. Erlbaum Associates.

*Cole, M. (2014). Speaking to read: Meta-analysis of peer-mediated learning for English language learners. Journal of Literacy Research, 46(3), 358-382. https://doi.org/10.1177/1086296X14552179

*Cole, M. (2018). Effectiveness of peer-mediated learning for English language learners: A meta-analysis. Research Ideas and Outcomes, 4, 1-77. https://doi.org/10.3897/rio.4.e29375

Cowan, R. (2008). The teacher's grammar of English with answers: A course book and reference guide. Cambridge University Press.

*Davis, D. (2010). A meta-analysis of comprehension strategy instruction for upper elementary and middle school students [Doctoral dissertation, Vanderbilt University]. Vanderbilt University Institutional Repository.

*de Vos, J., Schriefers, H., Nivard, M., \& Lemhöfer, K. (2018). A meta-analysis and metaregression of incidental second language word learning from spoken input. Language Learning, 68(4), 906-941. https://doi.org/10.1111/lang.12296

*Delgado, P., Vargas, C., Ackerman, R., \& Salmerón, L. (2018). Don't throw away your printed books: A meta-analysis on the effects of reading media on reading comprehension. Educational Research Review, 25, 23-38. https://doi.org/10.1016/j.edurev.2018.09.003

*Donker, A., de Boer, H., Kostons, D., Dignath-van Ewijk, C., \& van der Werf, M. (2014). Effectiveness of learning strategy instruction on academic performance: A meta-analysis. Educational Research Review, 11, 1-26. https://doi.org/10.1016/j.edurev.2013.11.002

*Elleman, A., Lindo, E., Morphy, P., \& Compton D. (2009). The impact of vocabulary instruction on passage-level comprehension of school-age children: A Meta-analysis. Journal of Research on Educational Effectiveness, 2, 1-44. https://doi.org/10.1080/19345740802539200

Enders, A. (2012). Management competence: Resource-based management and plant performance. Springer-Verlag.

*Fitton, L., McIlraith, A., \& Wood, C. (2018). Shared book reading interventions with English Learners: A $\begin{array}{lllll}\text { meta-analysis. Review of } & \text { 712-751. }\end{array}$ https://doi.org/10.3102/0034654318790909

Gambrell, L., Malloy, J., \& Mazzoni, S. (2011). Evidence-based best practices in comprehensive literacy instruction. In L. Morrow \& L. Gambrell (Eds.), Best practices in literacy instruction (pp. 11-36). The Guilford Press.

Glass, G. (1976). Primary, secondary, and meta-analysis of research. Educational Researcher, 5(10), 3-8. https://doi.org/10.3102/0013189X005010003

Glass, G. (1977). Integrating findings: The meta-analysis of research. Review of Research in Education, 5, 351-379. https://doi.org/10.2307/1167179

Goh, C. (2018). Learning speaking. In Burns, A. \& Richards, J. (Eds.), The Cambridge guide to learning English as a second language (pp. 204-212). Cambridge University Press.

*Goldberg, A., Russell, M., \& Cook, A. (2003). The effect of computers on student writing: A meta-analysis of studies from 1992 to 2002. The Journal of Technology, Learning, and Assessment, 2(1), 1-51. https://doi.org/10.1016/S8755-4615(02)00175-5

*Graham, S., \& Hebert, M. (2011). Writing to read: A meta-analysis of the impact of writing and writing instruction on reading. Harvard Educational Review, 81(4), 710-744. https://doi.org/10.17763/haer.81.4.t2k0m13756113566

*Graham, S., \& Perin, D. (2007). A meta-analysis of writing instruction for adolescent students. Journal of Educational Psychology, 99(3), 445-476. https://doi.org/10.1037/0022-0663.99.3.445 
*Graham, S., \& Sandmel, K. (2011). The process writing approach: A meta-analysis. Journal of Educational Research, 104, 396-407. https://doi.org/10.1080/00220671.2010.488703

*Graham, S., \& Santangelo, T. (2014). Does spelling instruction make students better spellers, readers, and writers? A meta-analytic review. Reading and Writing, 27, 1703-1743. https://doi.org/10.1007/s11145-014-9517-0

*Graham, S., Hebert, M., \& Harris K. (2015). Formative assessment and writing: A meta-analysis. The Elementary School Journal, 115(4), 523-547. https://doi.org/10.1086/681947

*Graham, S., Liu, K., Aitken, A., Ng, C., Bartlett, B., Harris, K. R., \& Holzapel, J. (2017). Balancing reading and writing instruction: A meta-analysis. Reading Research Quarterly, 53(3), 279-304. https://doi.org/10.1002/rrq.194

*Graham, S., Liu, X., Bartlett, B., Ng, C., Harris, K., Aitken, A., .... Talukdar, J. (2017). Reading for writing: A meta-analysis of the impact of reading and reading instruction on writing. Review of Educational Research, 88(2), 243-284. https://doi.org/10.3102/0034654317746927

*Graham, S., McKeown, D., Kiuhara, S., \& Harris, K. R. (2012). A meta-analysis of writing instruction for students in the elementary grades. Journal of Educational Psychology, 104(4), 879-896. https://doi.org/10.1037/a0029185

*Grgurovic, M., Chapelle, C., \& Shelley, M. (2013). A meta-analysis of effectiveness studies on computer

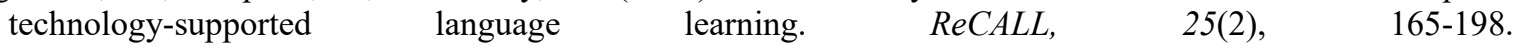
https://doi.org/10.1017/S0958344013000013

*Hall, C., Roberts, G., Cho, E., McCulley, L., Carroll, M., \& Vaughn, S. (2017). Reading instruction for English learners in the middle grades: A meta-analysis. Educational Psychology Review, 29, 763-794. https://doi.org/10.1007/s10648-016-9372-4

*Hall, M., \& Burns, M. (2018). Meta-analysis of targeted small-group reading interventions. Journal of School Psychology, 66, 54-66. https://doi.org/10.1016/j.jsp.2017.11.002

Hattie, J. (2009). A synthesis of over 800 meta-analyses relating to achievement. Routledge.

Hedges, L., \& Olkin, I. (1985). Statistical methods for meta-analysis. Academic Press.

Hollingsworth, J., \& Ybarra, S. (2013). Explicit direct instruction for English learners. Corwin.

*Huang, S. (2010). Effects of tasks and glosses on L2 incidental vocabulary learning: Meta-analyses [Doctoral dissertation, Texas A \& M University]. Texas A \& M University Libraries.

*Huisman, B., Saab, N., Broek, P., \& Driel, J. (2019). The impact of formative peer feedback on higher education students' academic writing: a Meta-analysis. Assessment \& Evaluation in Higher Education, 44(6), 863-880. https://doi.org/10.1080/02602938.2018.1545896

*Jeon, E., \& Day, R. (2016). The effectiveness of ER on reading proficiency: A meta-analysis. Reading in a Foreign Language, 28(2), 246-265.

*Kang, E., \& Han, Z. (2015). The efficacy of written corrective feedback in improving L2 written accuracy: A meta-analysis. The Modern Language Journal, 99(1), 1-18. https://doi.org/10.1111/modl.12189

*Kang, T. (2015). The effectiveness of multiple media tools in L2 listening: A meta-analysis. In V. Turel (Ed.), Intelligent design of interactive multimedia listening software (pp. 246-274). Information Science Reference. https://doi.org/10.4018/978-1-4666-8499-7.ch010

*Kansizoğlu, H. B. (2017). The effect of graphic organizers on language teaching and learning areas: A meta-analysis study. Education \& Science, 42(191), 139-164. https://doi.org/10.15390/EB.2017.6777

*Kao, C. (2013). Effects of focused feedback on the acquisition of two English articles. The Electronic Journal for English as a Second Language, 17(1), 1-15.

*Kao, C. (2014). The effects of digital game-based learning task in English as a foreign language contexts: A meta-analysis. Education Journal, 42(2), 113-141.

*Kao, C., \& Wible, D. (2014). A meta-analysis on the effectiveness of grammar correction in second language writing. English Teaching \& Learning, 38(3), 29-69.

Khader, F. (2012). Teachers' pedagogical beliefs and actual classroom practices in social studies instruction. American International Journal of Contemporary Research, 2(1), 73-92.

*Koster, M., Tribushinina, E., De Jong, P., \& van den Bergh, H. (2015). Journal of writing research, 7(2), 299-324. https://doi.org/10.17239/jowr-2015.07.02.2 
*Lee, J., Jang, J., \& Plonsky, L. (2015). The effectiveness of second language pronunciation instruction: A meta-analysis. Applied Linguistics, 36(3), 345-366. https://doi.org/10.1093/applin/amu040

*Lee, S.-K., \& Huang, H.-T. (2008). Visual input enhancement and grammar learning: A meta-analytic review. Studies in Second Language Acquisition, 30, 307-331. https://doi.org/10.1017/S0272263108080479

Li, L. (2017). New technologies and language learning. Palgrave. https://doi.org/10.1057/978-1-137-51768-5

*Liao, Y. K., Chang, L. H., \& Chang, C. C. (2010). Game-based learning vs. traditional instruction: A meta-analysis of thirty-eight studies from Taiwan. In C. Maddux (Ed.), Research highlights in information technology and teacher education (pp. 1491-1498). Society for Information Technology and Teacher Education.

*Lin, H. (2014a). Computer-mediated communication (CMC) in L2 oral proficiency development: A meta-analysis. ReCALL, 27(3), 261-287. https://doi.org/10.1017/S095834401400041X

*Lin, H. (2014b). Establishing an empirical link between computer-mediated communication (CMC) and SLA: A meta-analysis of the research. Language Learning \& Technology, 18(3), 120-147.

*Lin, W.-C., Huang, H.-T., \& Liou, H.-C. (2013). The effects of text-based SCMC on SLA: A meta analysis. Language Learning \& Technology, 17(2), 123-142.

Lipsey, M., \& Wilson, D. (1993). The efficacy of psychological, educational, and behavioral treatment: Confirmation from meta-Analysis. American Psychologist, 48(12), 1181-1209. https://doi.org/10.1037/0003-066X.48.12.1181

Littell, J., Corcoran, J., \& Pillai, V. (2008). Systematic Reviews and Meta-Analysis. Oxford University Press. https://doi.org/10.1093/acprof:oso/9780195326543.001.0001

*Little, C., Clark, J., Tani, N., \& Conner, C. (2018). Improving writing skills through technology-based instruction: A meta-analysis. Review of Education, 6(2), 183-201. https://doi.org/10.1002/rev3.3114

*Liu, J., \& Zhang, J. (2018). The effects of extensive reading on English vocabulary learning: A meta-analysis. English Language Teaching, 11(6), 1-15. https://doi.org/10.5539/elt.v11n6p1

*Ludwig, C., Guo, K., \& Georgiou, G. (2019). Are reading Interventions for English Language Learners Effective? A meta-analysis. Journal of Learning Disabilities, 52(3), 220-231. https://doi.org/10.1177/0022219419825855

*Lyster, R., \& Saito, K. (2010). Oral feedback in classroom SLA: A meta-analysis. Studies in Second Language Acquisition, 32, 265-302. https://doi.org/10.1017/S0272263109990520

Mackey, A., \& Goo, J. (2007). Interaction research in SLA: A meta-analysis and research synthesis. In A. Mackey (Ed.), Conversational interaction in second language acquisition: A collection of empirical studies (pp. 407-453). Oxford University Press.

*Maeng, U. (2014). The effectiveness of reading strategy instruction: A meta-analysis. English Teaching, 69(3), 105-127. https://doi.org/10.15858/engtea.69.3.201409.105

*Mahdi, S. H. (2018). Effectiveness of mobile devices on vocabulary learning: A meta-analysis. Journal of Educational Computing Research, 56(1), 134-154. https://doi.org/10.1177/0735633117698826

*Marulis, L. M., \& Neuman, S. B. (2010). The effects of vocabulary intervention on young children's word learning: A meta-analysis. Review of Educational Research, 80(3), 300-335. https://doi.org/10.3102/0034654310377087

Marzano, R. J. (1998). A theory-based meta-analysis of research on instruction. Mid-Continent Regional Educational Lab.

*Means, B., Toyama, Y., Murphy, R., \& Baki, M. (2013). The effectiveness of online and blended learning: A meta-analysis of the empirical literature. Teachers College Record, 115(3), 1-47.

*Mizumoto, A., \& Chujo, K. (2015). A meta-analysis of data-driven learning approach in the Japanese EFL classroom. English Corpus Studies, 22, 1-18.

*Nakanishi, T. (2015). A meta-analysis of extensive reading research. TESOL Quarterly, 49(1), 6-37. https://doi.org/10.1002/tesq.157

Nielson, K. (2016). Mobile-assisted language learning: Research-based best practices for maximizing learner success. In D. Mentor (Ed.), Handbook of research on mobile learning in contemporary classrooms (pp. 69-93). IGI Global. https://doi.org/10.4018/978-1-5225-0251-7.ch004

Norris, J., \& Ortega, L. (2000). Effectiveness of L2 instruction: A research synthesis and quantitative meta-analysis. Language Learning, 50(3), 417-528. https://doi.org/10.1111/0023-8333.00136 
Oxford, R. (2013). Teaching and researching: Language learning strategies. Routledge. https://doi.org/10.4324/9781315838816

Oxford, R. (2017). Teaching and researching language learning strategies: Self-regulation in context (2nd ed.). Routledge. https://doi.org/10.4324/9781315719146

Oxford, R., Lavine, R., \& Amerstorfer, C. (2018). Understanding language learning strategies: An innovative, complexity-based approach. In R. Oxford \& C. Amerstorfer (Eds.), Language learning strategies and individual learner characteristics: Situating strategy use in diverse contexts (pp. 5-29). Bloomsbury.

*Perez, M., Noortgate, W., \& Desmet, P. (2013). Captioned video for L2 listening and vocabulary learning: A meta-analysis. System, 41(3), 720-739. https://doi.org/10.1016/j.system.2013.07.013

*Plonsky, L. (2011). The Effectiveness of Second Language Strategy Instruction: A Meta-analysis. Language Learning, 61(4), 993-1038. https://doi.org/10.1111/j.1467-9922.2011.00663.x

*Puzio, K., \& Colby, G. (2010). The effects of within class grouping on reading achievement: A meta-analytic synthesis. Society for Research on Educational Effectiveness.

Rief, S., \& Stern, J. (2010). The dyslexia checklist: A Practical reference for parents and teachers. Jossey-Bass.

Roberts, B., Kuncel, N., Viechtbauer, W., \& Bogg, T. (2017). Meta-analysis in personality psychology: A primer. In R. Robins, R. Fraley \& R. Krueger (Eds.), Handbook of research methods in personality psychology (pp. 652-672). Guilford.

Sandelowski, M., \& Barroso, J. (2007). Handbook for synthesizing qualitative research. Springer.

Schmidt, F., \& Hunter, J. (2015). Methods of meta-Analysis: Correcting error and bias in research findings (3rd ed.). Sage. https://doi.org/10.4135/9781483398105

Schmitt, M. (2000). Vocabulary in language teaching. Cambridge University Press.

*Shintani, N. (2015). The effectiveness of processing instruction and production-based instruction on L2 grammar acquisition: A meta-analysis. Applied Linguistics, 36(3), 306-325. https://doi.org/10.1093/applin/amu067

*Shintani, N., Li, S., \& Ellis, R. (2013). Comprehension-based versus production-based grammar instruction: A meta-analysis of comparative studies. Language Learning, 63(2), 296-329. https://doi.org/10.1111/lang.12001

*Soe, K., Koki, S., \& Chang, J. (2000). Effect of computer-assisted instruction (CAI) on reading achievement: A meta-analysis. Pacific Resources for Education and Learning.

Stanley, G. (2013). Language learning with technology: Ideas for integrating technology in the classroom. Cambridge Press.

*Stockard, J., Wood, T., Coughlin, C., \& Khoury, C. (2018). The effectiveness of direct instruction curricula: A meta-analysis of a half century of research. Review of Educational Research, 20(10), 1-29. https://doi.org/10.3102/0034654317751919

*Sung, Y., Chang, K., \& Liu, T. (2016). The effects of integrating mobile devices with teaching and learning on students' learning performance: A meta-analysis and research synthesis. Computers \& Education, 94, 252-275. https://doi.org/10.1016/j.compedu.2015.11.008

*Sung, Y., Chang, K., \& Yang, J. (2015). How effective are mobile devices for language learning? A meta-analysis. Educational Research Review, 16, 68-84. https://doi.org/10.1016/j.edurev.2015.09.001

*Swanson, E., Stevens, E. A., Scammacca, N., Capin, P., Stewart, A., \& Austin, C. (2017). The impact of tier 1 reading instruction on reading outcomes for students in Grades 4-12: A meta-analysis. Reading and Writing, 30, 1639-1665. https://doi.org/10.1007/s11145-017-9743-3

Tafazoli, D., \& Romero, M. (2017). Multiculturalism and technology-enhanced language learning. IGI Global. https://doi.org/10.4018/978-1-5225-1882-2

*Taj, I., Sulan, N., Sipra, M., \& Ahmad, W. (2016). Impact of mobile assisted language learning (MALL) on EFL: A meta-analysis. Advances in Language and Literary Studies, 7(2), 76-83. https://doi.org/10.7575/aiac.alls.v.7n.2p.76

*Taylor, A. (2006). The effects of CALL versus traditional L1 glosses on L2 reading comprehension. CALICO Journal, 23(2), 309-318. https://doi.org/10.1558/cj.v23i2.309-318

*Taylor, A. (2014). L1 glossing and strategy training for improving L2 reading comprehension: a meta-analysis. International Journal of Quantitative Research in Education, 2(1), 39-68. https://doi.org/10.1504/IJQRE.2014.060973 
*Therrien, W. J. (2004). Fluency and comprehension gains as a result of repeated reading: A meta-analysis. Remedial and Special Education, 25(4), 252-261. https://doi.org/10.1177/07419325040250040801

*Thirakunkovit, S., \& Chamcharatsri, B. (2019). A meta-analysis of effectiveness of teacher and peer feedback: Implications for writing instructions and research. Asian EFL Journal, 21(1), 140-170.

*Truscott, J., (2007). The effect of error correction on learners' ability to write accurately. Journal of Second Language Writing, 16, 255-272. https://doi.org/10.1016/j.jslw.2007.06.003

*Tsai, Y., \& Tsai, C. (2018). Digital game-based second-language vocabulary learning and conditions of research designs: A meta-analysis study. Computers \& Education, 125, 345-357. https://doi.org/10.1016/j.compedu.2018.06.020

Ur, P. (2012). Grammar teaching: Theory, practice and English teacher education. In J. Hüttner, B. Mehlmauer-Larcher, S. Reichl \& B. Schiftner (Eds.), Theory and practice in EFL teacher education: Bridging the gap (pp. 83-100). Multilingual Matters. https://doi.org/10.21832/9781847695260-008

Uther, M. (2019). Mobile learning: Trends and practices. In M. Uther (Ed.), Mobile learning (pp. 1-3). MDPI. https://doi.org/10.3390/educsci9010033

*Vahedi, V., Ghonsooly, B., \& Pishghadam, R. (2016). Vocabulary glossing: A meta-analysis of the relative effectiveness of different gloss types on L2 vocabulary acquisition. Teaching English with Technology, $16(1), 3-25$.

*Vo, H., Zhu, C., \& Diep, N. (2017). The effect of blended learning on student performance at course-level in higher education: A meta-analysis. Studies in Educational Evaluation, 53, 17-28. https://doi.org/10.1016/j.stueduc.2017.01.002

*Wang, L. (2014). Meta-analysis of peer-peer interaction in L2 English speaking assessment. English Teaching \& Learning, 38(3), 103-137.

White, C. (2008). Language learning strategies in independent language learning: An overview. In S. Hurd \& T. Lewis (Eds.), Language learning strategies in independent settings (pp. 3-24). Multilingual Matters. https://doi.org/10.21832/9781847690999-003

*Won, M. (2008). The effects of vocabulary instruction on English language learners: A meta-analysis [Doctoral dissertation, Texas Tech University]. Texas Tech University Libraries.

*Xu, Z., Banerjee, M., Ramirez, G., Zhu, G., \& Wijekumar, K. (2019). The effectiveness of educational technology applications on adult English language learners' writing quality: a meta-analysis. Computer Assisted Language Learning, 32(1-2), 132-162. https://doi.org/10.1080/09588221.2018.1501069

*Yan, X., Maeda, Y., Lv, J., \& Ginther, A. (2016). Elicited imitation as a measure of second language proficiency: A narrative review and meta-analysis. Language Testing, 33(4), 497-528. https://doi.org/10.1177/0265532215594643

*Yousefi, M., \& Biria, R. (2018). The effectiveness of L2 vocabulary instruction: a meta-analysis. Journal of Second and Foreign Language Education, 3(21). https://doi.org/10.1186/s40862-018-0062-2

*Yun, J. (2011). The effects of hypertext glosses on L2 vocabulary acquisition: a meta-analysis. Computer Assisted Language Learning, 24(1), 39-58. https://doi.org/10.1080/09588221.2010.523285

*Zhao, Y. (2003). Recent developments in technology and language learning: A literature review and meta-analysis. CALICO Journal, 21(1), 7-27. https://doi.org/10.1558/cj.v21i1.7-27

Zhao, Y. (2005). Research in technology and second language learning: Developments and directions. Information Age Publishing.

*Ziegler, N. (2015). Synchronous computer-mediated communication and interaction: A meta-analysis. Studies in Second Language Acquisition, 38, 553-586. https://doi.org/10.1017/S027226311500025X

\section{Copyrights}

Copyright for this article is retained by the author(s), with first publication rights granted to the journal.

This is an open-access article distributed under the terms and conditions of the Creative Commons Attribution license (http://creativecommons.org/licenses/by/4.0/). 